\title{
Atomic gas fractions in active galactic nucleus host galaxies
}

\author{
Sara L. Ellison ${ }^{1}$, Toby Brown ${ }^{2}$, Barbara Catinella ${ }^{3,4}$, Luca Cortese Ca $^{3,4}$ \\ ${ }^{1}$ Department of Physics $\mathcal{E}$ Astronomy, University of Victoria, Finnerty Road, Victoria, British Columbia, V8P 1A1, Canada \\ ${ }^{2}$ Department of Physics $\mathcal{G}$ Astronomy, McMaster University, 1280 Main St. W., Hamilton, Ontario L8S 4M1, Canada \\ ${ }^{3}$ International Centre for Radio Astronomy Research, The University of Western Australia, 35 Stirling Hwy, Crawley, WA 6009, Australia \\ ${ }^{4}$ ARC Centre of Excellence for All Sky Astrophysics in 3 Dimensions (ASTRO 3D)
}

22 November 2018

\begin{abstract}
The feedback from an active galactic nucleus (AGN) is frequently invoked as a mechanism through which gas can be heated or removed from a galaxy. However, gas fraction measurements in AGN hosts have yielded mixed support for this scenario. Here, we re-visit the assessment of $f_{\text {gas }}\left(=\mathrm{M}_{H I} / \mathrm{M}_{\star}\right)$ in $\mathrm{z}<0.05$ AGN hosts in the Sloan Digital Sky Survey (SDSS) using two complementary techniques. First, we investigate $f_{\text {gas }}$ for 75 AGN host galaxies in the extended GALEX Arecibo SDSS Survey (xGASS), whose atomic gas fractions are complete to a few percent. Second, we construct H i spectral stacks of 1562 AGN from the Arecibo Legacy Fast ALFA (ALFALFA) survey, which enables us to extend the AGN sample to lower stellar masses. Both techniques find that, at fixed $\mathrm{M}_{\star}$, AGN hosts with $\log \left(\mathrm{M}_{\star} / \mathrm{M}_{\odot}\right) \gtrsim 10.2$ are $\mathrm{H}_{\mathrm{I}}$ rich by a factor of $\sim 2$. However, this gas fraction excess disappears when the control sample is additionally matched in star formation rate (SFR), indicating that these AGN hosts are actually $\mathrm{H}_{\mathrm{i}}$ normal. At lower stellar mass, the stacking analysis reveals that AGN hosts are $\mathrm{H}_{\mathrm{I}}$ poor at fixed stellar mass. In the lowest $\mathrm{M}_{\star}$ regime probed by our sample, $9<\log$ $\left(\mathrm{M}_{\star} / \mathrm{M}_{\odot}\right)<9.6$, the H I deficit in AGN hosts is a factor of $\sim 4$, and remains at a factor of $\sim 2$ even when the control sample is additionally matched in SFR. Our results help reconcile previously conflicting results, by showing that matching control samples by more than just stellar mass is critical for a rigourous comparison.
\end{abstract}

Key words: galaxies: active, galaxies: ISM, galaxies: Seyfert

\section{INTRODUCTION}

Active galactic nuclei (AGN) have been widely proposed as an effective pathway for shutting down star formation in galaxies, as they provide an energy mechanism through which gas can be either heated or removed (see Harrison et al. 2018 for a recent review). Indirect observational evidence for the role of AGN in quenching star formation comes from the connection between the likelihood that a galaxy is no longer forming stars and the properties of galaxy centres', such as bulge fraction (Omand et al. 2014), bulge mass (Bluck et al. 2014; Lang et al. 2014), Sersic index (Wuyts et al. 2011; Mendel et al. 2013), central stellar mass density (Cheung et al. 2012; Fang et al. 2013; Woo et al. 2015), central velocity dispersion (Bluck et al. 2016; Teimoorinia et al. 2016) and black hole mass (Terrazas et al. 2016). However, it has been argued that such a 'morphology quiescence' relation need not require a causal connection between an AGN and the cessation of star formation (Lilly \& Carollo 2016).

Conclusive observational evidence for AGN driven quenching has remained elusive, with apparently conflicting results in the literature. For example, although local radio-selected AGN are hosted by passive elliptical galaxies (e.g. Best \& Heckman 2012), which could be interpreted as a causal relationship between nuclear activ- ity and the shut-down of star formation, most AGN are found in star-forming galaxies (e.g. Hughes \& Cortese 2009; Santini et al. 2012; Rosario et al. 2013). Indeed, AGN selected in the mid-infra red (IR) actually show elevated star formation rates (Shimizu et al. 2015; Ellison et al. 2016b; Cowley et al. 2016; Azadi et al. 2017). Linking AGN to quenching via their star formation rate (SFR) therefore seems to be highly dependent on the method through which the AGN are selected.

An alternative approach to linking feedback processes with quenching is through the study of gas flowing out of the galaxy and outflows are indeed common in AGN host galaxies (e.g. Mullaney et al. 2013; McElroy et al. 2015; Woo et al. 2016). However, there is significant complexity (see e.g. the arguments presented in Harrison et al 2018) in linking the evident outflows with the eventual fate of its constituent gas: Although large masses may be entrained in these outflows, the gas might not actually escape the galaxy's potential well, and may eventually become available for star formation once again. Indeed, although some studies have proposed that massive outflows could play an important role in quenching (e.g. Cicone et al. 2014; Baron et al. 2018), other studies have concluded that most AGN-driven winds have velocities well below the escape value and that low mass fractions are ejected (e.g. Concas et 
al. 2017; Davies et al. 2018; Fluetsch et al. 2018; Roberts-Borsani \& Saintonge 2018). Even the most powerful local sources (starbursts and quasars) do not seem adequate to actually remove a significant fraction of their gas reservoir (e.g. Pereira-Santaella et al. 2018; Shangguan, Ho \& Xie 2018). Simulations have similarly concluded that low redshift galaxies mostly recycle the material in their outflows, rather than ejecting it (e.g. Muratov et al. 2015; Angles-Alcazar et al. 2017).

In this study, we investigate the potential role of AGN in quenching star formation by assessing the impact of the AGN on the host galaxy's atomic gas reservoir. Again, the literature yields conflicting results on this topic. Some studies have found that the atomic gas fractions of AGN hosts are consistent with inactive galaxies (e.g. Fabello et al. 2011; Gereb et al. 2015), and a similar conclusion has been drawn from molecular gas fractions (e.g. Saintonge et al. 2012; 2017; Husemann et al. 2017; Rosario et al. 2018). However, other studies have found both elevated (e.g. Ho, Darling \& Greene 2008; Berg et al. 2018) and suppressed (Haan et al. 2008) $\mathrm{H}_{\mathrm{I}}$ gas reservoirs in and around AGN host galaxies.

There are several subtleties associated with these previous studies that may contribute to their conflicting conclusions. For example, Bradford et al. (2018) have recently suggested that the impact of AGN feedback could be mass dependent, since they find evidence of gas depletion only in the low mass galaxies of their sample. Moreover, several of these previous studies rely on relatively shallow H i data, which can bias our view of the 'norm' and often necessitates stacking. The use of deeper data and accounting for non-detections can significantly change the outcome of observational comparisons. For example, the Arecibo Legacy Fast ALFA (ALFALFA) survey (Giovanelli et al. 2005) is a large $\mathrm{H}_{\mathrm{I}}$ survey with relatively shallow depth. Using ALFALFA, and similar depth targeted observations, Ellison et al. (2015) found that recently merged galaxies had similar atomic gas fractions to control galaxies. However, repeating this experiment with much deeper $\mathrm{H}_{\mathrm{I}}$ data, and accounting carefully for non-detections, Ellison, Catinella $\&$ Cortese (2018) have recently shown that post-mergers are actually a factor of $\sim 3$ more $\mathrm{H}$ i rich than isolated galaxies of the same stellar mass.

In the work presented here, we use two different approaches to assess the $\mathrm{H}_{\mathrm{I}}$ gas fraction of AGN host galaxies. First, we use deep, targeted $21 \mathrm{~cm}$ measurements from the Galaxy Evolution Explorer (GALEX) Arecibo Sloan Digital Sky Survey (SDSS) Survey (xGASS, Catinella et al. 2018) and compare H i gas fractions of optically identified AGN host galaxies with a stellar mass and SFR matched control sample. However, although xGASS reaches atomic gas fractions $\left(f_{\text {gas }}=\mathrm{M}_{\mathrm{HI}} / \mathrm{M}_{\star}\right)$ as deep as a few percent, there are only 75 (optically selected) AGN in the xGASS sample. For a complementary analysis, we use the large, but shallow, ALFALFA survey (Giovanelli et al. 2005; Haynes et al. 2018). Since individual ALFALFA spectra are an order of magnitude less sensitive than xGASS, we use an $\mathrm{H}_{\mathrm{i}}$ spectral stacking approach to measure average atomic gas fractions for samples of AGN hosts, along with stellar mass and SFR matched control samples.

The paper is laid out as follows. In Sec. 2 we describe our sample selection and methods. In Sec. 3 we present our results for the two methods: gas fractions in individual xGASS galaxies (Sec. 3.1) and in ALFALFA spectral stacks (Sec. 3.2). A discussion is presented in Sec. 4 and a summary in Sec.5 We adopt a cosmology in which $\mathrm{H}_{0}=70 \mathrm{~km} / \mathrm{s} / \mathrm{Mpc}, \Omega_{M}=0.3, \Omega_{\Lambda}=0.7$.

\section{METHODS}

\subsection{Parent galaxy sample and AGN identification}

All of the galaxies used in our analysis are selected from the SDSS data release 7 (DR7). We make use of the public JHU/MPA catalog: 1 of stellar masses and emission line fluxes (e.g. Kauffmann et al. 2003a; Brinchmann 2004). We will also make use of SFRs in our analysis. Although a measurement of SFR is available in the MPA/JHU catalogs, based on the strength of the D4000 break (Brinchmann et al. 2004), these can have large uncertainties for non-star-forming and AGN galaxies (e.g. Rosario et al. 2016). We therefore use SFRs obtained from the UV+optical stellar spectral energy distribution (SED) fits (no AGN) in the GALEXSDSS-Wide-field Infra-red Survey Explorer (WISE) Legacy Cata$\log$ (GSWLC2, Salim et al. 2016), using the A2 catalog of Salim, Boquien \& Lee (2018). Typical uncertainties on the stellar masses used in our work are $\sim 0.1$ dex (Kauffmann et al. 2003a). However, uncertainties on SFRs are highly dependent on the SFR itself; for star-forming galaxies uncertainties are typically $0.1 \mathrm{dex}$, but rise to values of $\sim 0.6$ dex for passive galaxies (see Fig. 6 in Salim et al. 2016).

To identify galaxies that host AGN we first apply the continuum and Balmer S/N cuts described in Scudder et al. (2012), and then correct emission line fluxes according to a Small Magellanic Cloud dust law as described by Pei (1992). We then impose a S/N cut of 5 in the four emission lines required for the traditional optical AGN classification: [NII], $\mathrm{H} \alpha$, [OIII], $\mathrm{H} \beta$, a cut which largely removes galaxies with shock and Low Ionization Nuclear Emission Region (LINER)-like spectra. The diagnostic separation of Kauffmann et al. (2003b) is used to distinguish AGN from galaxies whose emission spectra are dominated by star formation ${ }^{3}$ Specifically, a galaxy is classified as hosting an $\mathrm{AGN}$ if $\log ([\mathrm{OIII}] / \mathrm{H} \beta)>$ $0.61 /(\log ([\mathrm{NII}] / \mathrm{H} \alpha)-0.05)+1.3($ Eqn. 1 from Kauffmann et al. 2003b).

\section{$2.2 H_{\text {I }}$ measurements from xGASS}

In the first of the two analyses presented in this work, we compare the H I gas fractions of individual AGN hosts observed as part of the xGASS survey (Catinella et al. 2018). The xGASS sample contains $\sim 1200 \mathrm{z}<0.05$ galaxies with measurements of $f_{\text {gas }}$ over a mass range $9.0<\log \left(\mathrm{M}_{\star} / M_{\odot}\right)<11.5$. $\mathrm{H}_{\mathrm{I}}$ gas masses are derived in the standard way from $21 \mathrm{~cm}$ fluxes, e.g. Eqn 1 of Catinella et al. (2010), which can be combined with stellar masses to obtain gas fractions: $f_{\text {gas }}=\mathrm{M}_{\mathrm{HI}} / \mathrm{M}_{\star}$. We use both detections and nondetections in the final xGASS release but reject any xGASS detections identified in the survey's catalog to have possible confusion from neighbouring sources. Typical uncertainties on $\mathrm{H}_{\text {I }}$ gas masses in xGASS are 0.05 dex.

In Fig. 1 we show the distribution of $\mathrm{H}_{\mathrm{I}}$ gas fractions for the xGASS sample, showing galaxies identified as hosting AGN as star symbols. Downward pointing arrows indicate $\mathrm{H}_{\mathrm{I}}$ upper limits. There are 75 optically-selected AGN in the xGASS sample, of which 65 are $\mathrm{H}_{\mathrm{I}}$ detections. There are 887 non-AGN in the sample (which includes both emission line galaxies not classified as AGN,

\footnotetext{
1 https://wwwmpa.mpa-garching.mpg.de/SDSS/

2 http://pages.iu.edu/ ssalims/gswlc/

3 All of the trends presented in this paper are recovered if we use instead the Kewley et al. (2001) AGN selection criteria.

${ }^{4}$ http://xgass.icrar.org/
} 


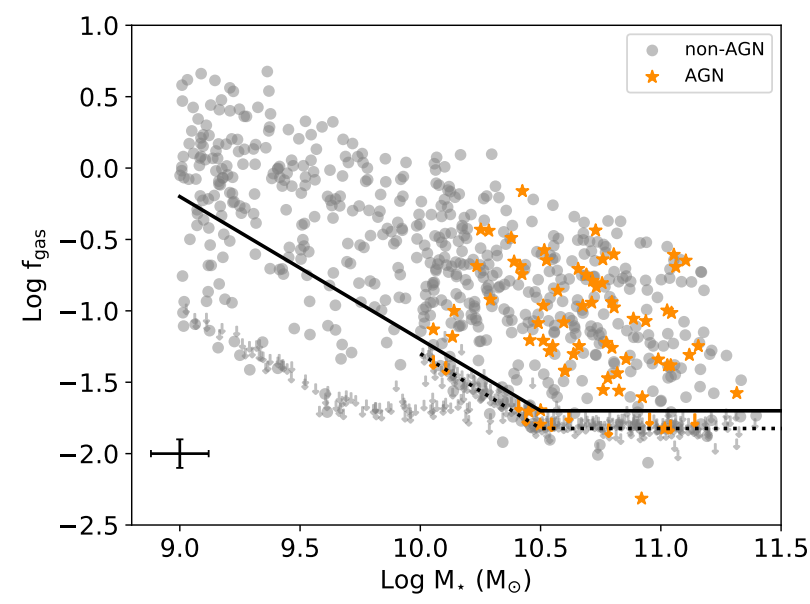

Figure 1. H i gas fractions as a function of stellar mass for AGN (orange stars) and non-AGN (grey circles) host galaxies in the xGASS survey. For both samples, H I non-detections are shown by downward pointing arrows. The dotted line shows the detection threshold of the GASS survey $\left(\log \left[\mathrm{M}_{\star}\right]\right.$ $\left.\mathrm{M}_{\odot}\right]>10$ ), with our modified threshold shown by the solid line that extends to lower masses more conservatively separates the detections from the upper limits. The modified detection threshold corresponds to $f_{\text {gas }}<2$ percent for $\log \left(\mathrm{M}_{\star} / M_{\odot}\right)>10.5$ and $\log \mathrm{M}_{\mathrm{HI}}=8.8 \mathrm{M}_{\odot}$ below that mass. Typical uncertainties are shown by the errorbar in the lower left.

as well as galaxies without strong emission lines), of which 568 are $\mathrm{H}_{\mathrm{i}}$ detections. Fig. 1 shows the well known bias that the optically selected AGN in the sample occur at relatively high masses (e.g. Kauffmann et al. 2003b). The dotted line in Fig. 1 shows the original survey's goal detection threshold (Catinella et al. 2010). However, following Ellison et al. (2018), we make a slightly more conservative demarcation between detections and non-detections corresponding to $f_{\text {gas }}<2$ percent for $\log \left(\mathrm{M}_{\star} / M_{\odot}\right)>10.5$ and $\log$ $\mathrm{M}_{\mathrm{HI}}=8.8 \mathrm{M}_{\odot}$ below that mass (solid line in Fig. 1). The exact detection threshold definition does not affect our results. Detections below the adopted threshold are counted as non-detections for the statistics in the rest of this paper.

\subsection{Spectral stacking of ALFALFA data}

In this work, we use data cubes from the ALFALFA 100 percent sample (Haynes et al. 2018). The final data release of the ALFALFA blind-HI survey is the largest census of atomic gas content in the local Universe to date, mapping over 7000 square degrees of sky out to a redshift of $z \sim 0.06$.

The subsamples of galaxies used for our stacking analysis ( $\mathrm{Sec}$ 3.2 are drawn from a parent sample of 34,142 galaxies that is selected according to stellar mass $\left(9 \leqslant \log \left(\mathrm{M}_{\star} / \mathrm{M}_{\odot}\right) \leqslant 11.5\right)$ and redshift $(0.02 \leqslant z \leqslant 0.05)$ from the overlap in volume between the full ALFALFA survey and SDSS DR7. The increase in the number of galaxies between the parent sample used in this work and that of Brown et al. (2015) comes from our use of the recently available ALFALFA 100 percent datacubes.

After requiring that galaxies in the ALFALFA parent sample are also included in the GSWLC, and that there is an AGN (according to Kauffmann et al 2003b) classification available, our sample is reduced to 28,678 galaxies, which we refer to hereafter as 'the ALFALFA sample'. From this reduced sample, we identify 1562 optically-selected AGN, 509 of which are detected by ALFALFA. There are thus 27,116 non-AGN host galaxies in the ALFALFA

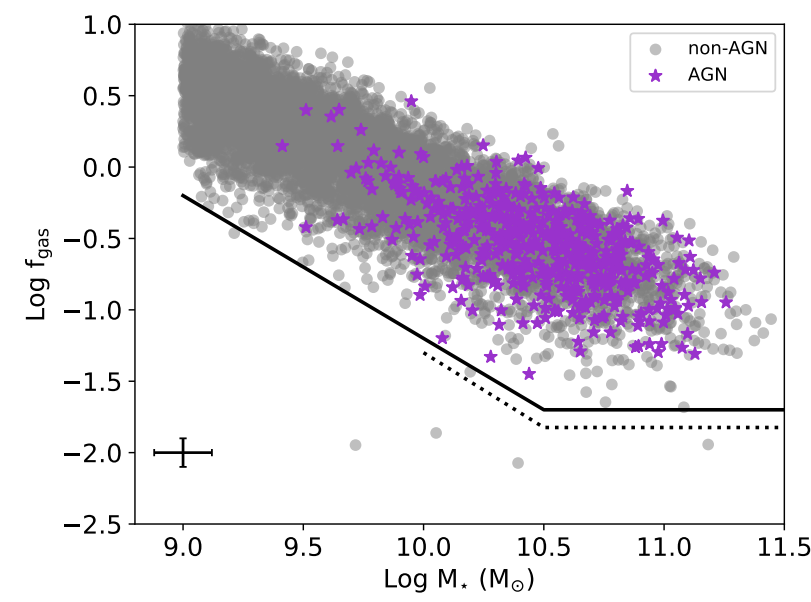

Figure 2. H I gas fractions as a function of stellar mass for AGN (purple stars) and non-AGN (grey circles) host galaxies in the ALFALFA sample. Only $\mathrm{H}$ I detections are available in the ALFALFA catalog. The axis ranges and solid and dashed lines (xGASS detection thresholds) are the same as shown in Fig. 1 for ease of comparison between the two surveys. Typical uncertainties are shown by the errorbar in the lower left.

sample that can be used as a comparison sample, of which 7722 have $\mathrm{H}_{\mathrm{I}}$ detections.

In Fig. 2 we present the distribution of gas fractions for the individual $21 \mathrm{~cm}$ detections in the ALFALFA sample, taken directly from the public ALFALFA catalog. In contrast to xGASS (Fig. 1), no upper limit information is provided in the ALFALFA catalogs. The distribution of points in Fig. 2 therefore shows the ALFALFA sample's distribution of gas fractions for $\mathrm{H}_{\mathrm{I}}$ detections, but does not represent the full set of data included in our spectral stacks (which include non-detections as well, see below). As in Fig. 1. we plot AGN host galaxies as stars and non-AGN hosts as circles 5 We set the $\mathrm{x}$ - and $\mathrm{y}$-axis ranges of Fig. 2 (ALFALFA) to match that of Fig, 1 (xGASS), and also reproduce the xGASS detection thresholds in the figure, to facilitate comparison between the two surveys and demonstrate the relative depth of xGASS compared with ALFALFA. Fig. 2 shows that ALFALFA's H i detections are typically 0.5 dex shallower than the xGASS detection threshold at fixed stellar mass.

The ALFALFA analysis presented here does not use gas fractions from the public catalogs, but rather produces spectral stacks directly from the original data cubes. Following the steps outlined in Brown et al. (2015), we extract individual $\mathrm{H}_{\mathrm{I}}$ spectra for each galaxy using a $4 \times 4$ arcminute aperture and $5500 \mathrm{~km} \mathrm{~s}^{-1}$ velocity cut centered on its position in the ALFALFA datacubes. The $\mathrm{H}_{\mathrm{I}}$ stacking is performed by co-adding $21 \mathrm{~cm}$ spectra that are aligned in velocity (redshift) to yield an average atomic gas measurement for a given sample of galaxies regardless of whether they are individually detected in $\mathrm{H}_{\mathrm{I}}$. The stochastic nature of each individual $\mathrm{H}_{\mathrm{I}}$ spectrum's noise means that the signal-to-noise ratio of the stacked spectrum increases as function of the square root of the galaxies in each stack. Note that, following Brown et al. (2015), we weight the individual spectra in each stack by the galaxy's stellar mass and luminosity distance so that the final stacked spectrum is in 'gas frac-

\footnotetext{
5 Throughout this paper, we facilitate the visual comparison of the xGASS and ALFALFA analyses by using orange and purple symbols in figures that refer to these two samples, respectively.
} 
tion units'. For a complete description of our data processing and stacking methodology, we refer the reader to Brown et al. (2015).

\section{RESULTS}

\subsection{Gas fractions of AGN in the xGASS sample}

In Fig. 3 we show the fraction of $\mathrm{H}_{\mathrm{I}}$ detections in the xGASS sample as a function of stellar mass, with $1 \sigma$ bounds shown as the shaded regions. The AGN host $\mathrm{H}_{\mathrm{I}}$ detection fraction (shown in solid orange) is significantly above the non-AGN host $\mathrm{H}$ i detection fraction (shown in dashed grey) for most stellar masses. The higher detection fraction amongst the AGN host galaxies suggests that their gas fractions are systematically higher than their non-active counterparts at fixed stellar mass.

For a quantitative assessment of the difference in $f_{\text {gas }}$ between AGN and non-AGN hosts, we follow Ellison et al. (2018), and compute the $\mathrm{H}_{\mathrm{I}}$ gas fraction offset, $\Delta f_{\text {gas }} . \Delta f_{\text {gas }}$ is computed for each AGN host galaxy in turn by identifying all non-AGN galaxies in the xGASS sample of the same stellar mass (within \pm 0.15 dex) and computing the difference between $\log f_{\text {gas }}$ in the AGN galaxy and median value of the matched control galaxies. There are typically between $100-200$ non-AGN control galaxies matched to each AGN host.

The detection fraction in the non-AGN sample exceeds 50 per cent (horizontal dashed line in Fig. 3) for stellar masses log $\left(\mathrm{M}_{\star} / \mathrm{M}_{\odot}\right) \leqslant 10.8$ (vertical dashed line in Fig. 3). Therefore, the median gas fraction and $\Delta f_{\text {gas }}$ are well constrained in this mass regime, even accounting for non-detections. Of the 75 AGN in the full xGASS sample (Fig 1 ), 50 have $\log \left(\mathrm{M}_{\star} / \mathrm{M}_{\odot}\right) \leqslant 10.8$ and hence have reliable $\Delta f_{\text {gas }}$ determinations. Of these, 41 are $\mathrm{H}_{\mathrm{I}}$ detections (and are above the adopted detection threshold shown by the solid line in Fig. 1) and nine are non-detections (or detections below the solid line in Fig. 11.

Fig. 4 shows the distribution of $\Delta f_{\text {gas }}$ for the sample of 50 AGN hosts with $\log \left(\mathrm{M}_{\star} / \mathrm{M}_{\odot}\right) \leqslant 10.8$. The $\mathrm{H}_{\mathrm{I}}$ non-detections lead to upper limits in the calculation of $\Delta f_{\text {gas }}$; these nine galaxies are shown as the open histogram in Fig. 4 The median $\Delta f_{\text {gas }}$ of the full sample of 50 AGN hosts (including the nine upper limits) is +0.29 dex, representing a factor of two $f_{\text {gas }}$ enhancement on average at fixed stellar mass. In Fig. 5 we plot $\Delta f_{\text {gas }}$ versus total stellar mass. The enhanced $f_{\text {gas }}$ is fairly consistent across the full stellar mass range of our sample, although, intriguingly, the five lowest mass optical AGN hosts, with $10.0<\log \left(\mathrm{M}_{\star} / \mathrm{M}_{\odot}\right)<10.2$, do not show enhancements. We re-visit the mass dependence of $\Delta f_{\text {gas }}$ in the next Section, where the ALFALFA stacking analysis offers better statistics at lower stellar masses.

Although optically selected AGN have slightly lower SFRs than other main sequence galaxies (e.g. Ellison et al. 2016b; Leslie et al. 2016), they are nonetheless usually hosted by star-forming and 'green valley' galaxies, with a relative paucity amongst passive galaxies (e.g. Hughes \& Cortese 2009; Santini et al. 2012; Rosario et al. 2013). The elevated gas fractions in AGN hosts at fixed stellar mass may be a manifestation of this tendency to avoid the passive population, whereas non-AGN control galaxies have a wide range of SFR at a given stellar mass.

In Fig. 6 we investigate the tendency of AGN to be hosted in star forming galaxies in the xGASS sample by plotting the distribution of SFR vs. stellar mass for the xGASS non-AGN sample with grey circles and AGN hosts in xGASS in orange stars. Fig. 6 shows that, as expected, the AGN hosts in the xGASS sample

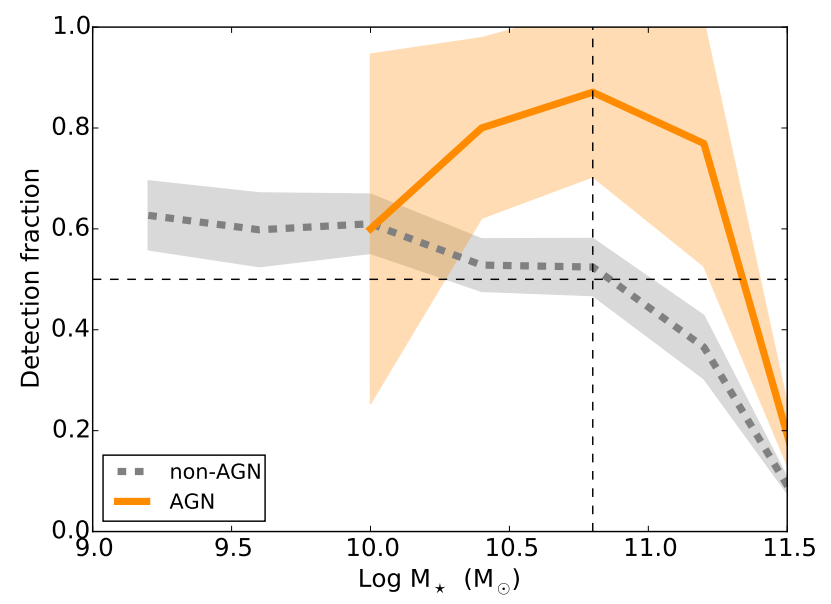

Figure 3. The H I detection fraction of AGN (solid orange line) and nonAGN (dashed grey line) host galaxies in the xGASS sample with $1 \sigma$ bounds shaded. The vertical dashed line corresponds to $\log \mathrm{M}_{\star}=10.8 \mathrm{M}_{\odot}$ above which the xGASS detection fraction drops below 50 per cent (horizontal dashed line).

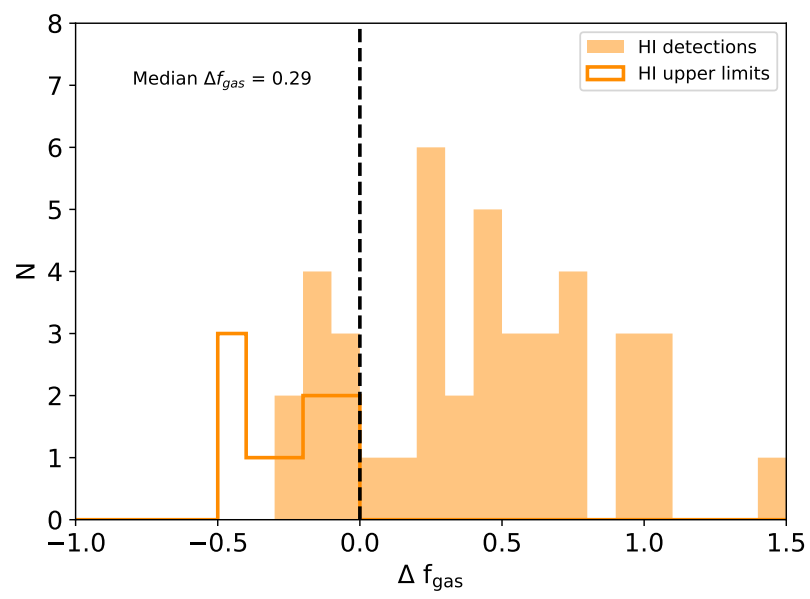

Figure 4. $\Delta f_{\text {gas }}$ distribution for $\mathrm{AGN}$ host galaxies, matched to control galaxies in $\mathbf{M}_{\star}$, in the xGASS sample with $\log \left(\mathrm{M}_{\star} / \mathrm{M}_{\odot}\right) \leqslant 10.8$. Both detections and upper limits are considered in the control sample. The solid/open histograms show $\Delta f_{\text {gas }}$ for $\mathrm{H}_{\text {I }}$ detections/upper limits respectively.

lie predominantly along the star-forming main sequence, with relatively few located in either the green valley or in the regime of 'quenched' galaxies. We therefore repeat our calculation of $\Delta f_{\text {gas }}$, but now match in both $M_{\star}$ and SFR (equivalent to matching in sSFR at fixed mass). The requirement of a measured SFR in the GSWLC slightly reduces the size of the AGN sample, to $39 \mathrm{H} \mathrm{I}$ detections and $9 \mathrm{HI}_{\mathrm{I}}$ non-detections (down from 41 and 9 in the original sample). We require that the SFRs of control galaxies be matched to that of the AGN to within \pm 0.1 dex. The additional requirement of a SFR match greatly reduces the number of controls for each AGN host, from more than 100 when only $M_{\star}$ is matched to typically 10-20 with matched SFR and $M_{\star}$.

In Figure 7 we show the distribution of $\Delta f_{\text {gas }}$ when SFR is included with $\mathrm{M}_{\star}$ in the matching criteria. In contrast to the mostly positive values in Figure 4 Figure 7 shows that the inclusion of 


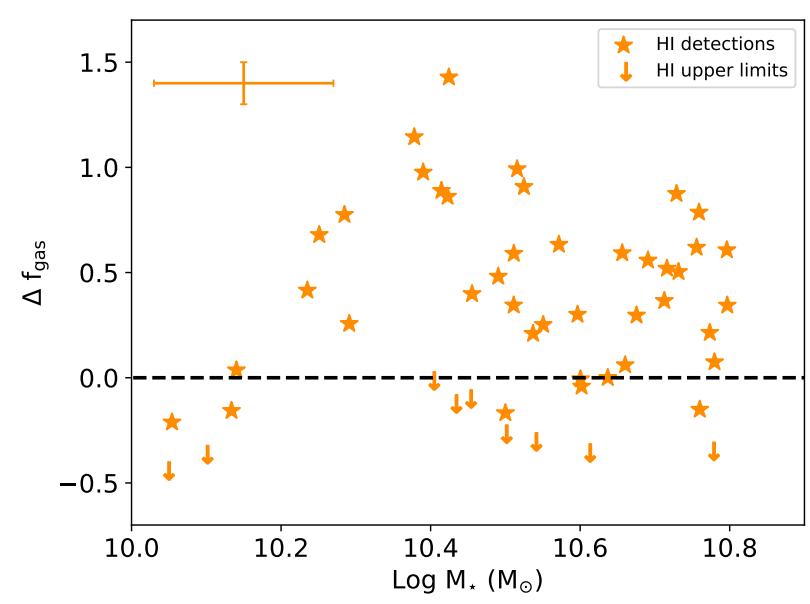

Figure 5. $\Delta f_{\text {gas }}$ as a function of stellar mass for AGN hosts in the xGASS sample. Solid stars indicate $\mathrm{H}_{\mathrm{I}}$ detections and downward pointing arrows are upper limits. Typical uncertainties are shown by the errorbar in the upper left.

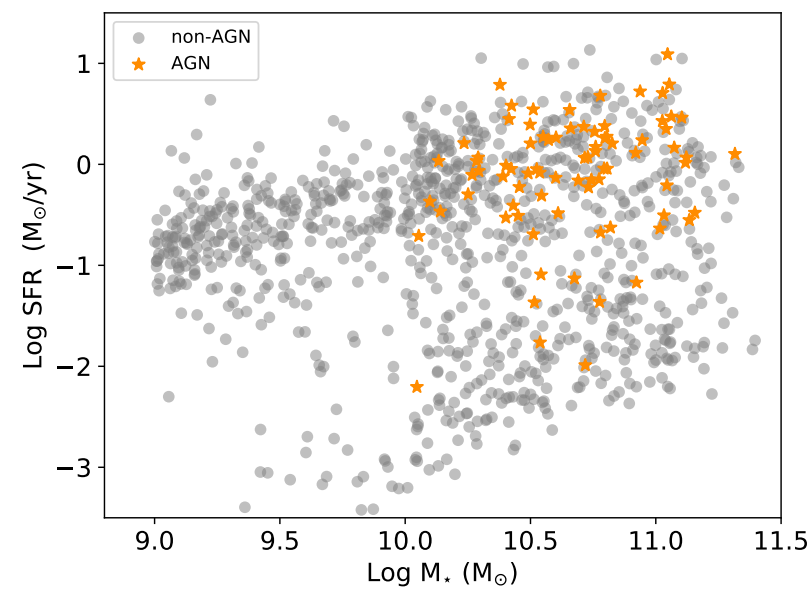

Figure 6. Distribution of SFR versus stellar mass for the xGASS sample. Non-AGN hosts are plotted with grey circles and AGN hosts in orange stars. AGN hosts tend to lie on the star forming main sequence, or green valley, with relatively few quenched hosts.

SFR in the matching process now yields a distribution of $\Delta f_{\text {gas }}$ that is more symmetric around zero. The median (including nondetections) is slightly negative $(-0.08 \mathrm{dex})$ but there is no statistically significant deviation from a gaussian distribution centred at zero.

We repeat the SFR matching analysis using SFRs from two other catalogs. The first alternative is using the SFRs published in the xGASS catalog by Janowiecki et al. (2017), who use GALEX and WISE photometry. Second, we determine SFRs from the total IR luminosities $\left(\mathrm{L}_{\mathrm{IR}}\right)$ obtained by the artificial neural network calibration of the Herschel Stripe 82 overlap (Ellison et al. 2016a) as a proxy for SFR, requiring $\sigma_{A N N} \leqslant 0.1$. We find qualitatively consistent distributions of $\Delta f_{\text {gas }}$ when using any of these three (Salim et al. 2018; Janowiecki et al. 2017; Ellison et al. 2016a) SFR indicators. In all cases, we find that once matched in both SFR and stellar mass, the AGN host gas fractions are consistent with those of the non-AGN control galaxies.

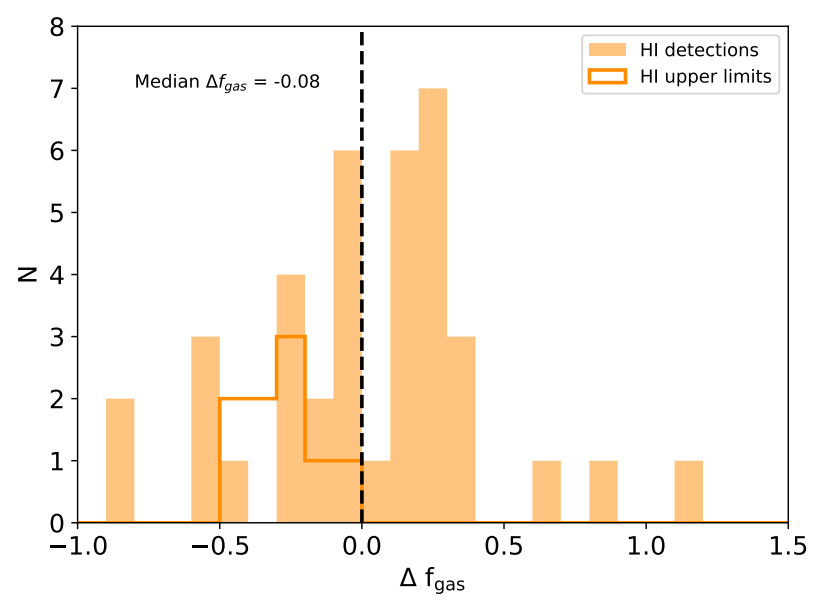

Figure 7. $\Delta f_{\text {gas }}$ distribution for AGN host galaxies matched in both $\mathrm{M}_{\star}$ and SFR for the xGASS sample with $\log \left(\mathrm{M}_{\star} / \mathrm{M}_{\odot}\right) \leqslant 10.8$ considering both detections and upper limits in the control sample. SFRs are taken from the GSWLC of Salim et al. (2016). The solid/open histograms show $\Delta f_{\text {gas }}$ for $\mathrm{H}_{\mathrm{I}}$ detections/upper limits respectively.

The results from this section show that comparing the AGN to a non-AGN control sample matched in stellar mass alone will yield mis-leading results. The gas fraction enhancement seen in Figs. 4 and 5 are purely the result of AGN preferentially being hosted in star forming galaxies. Once taking this 'bias' into account, there is no statistically significant difference between the $\mathrm{H}_{\mathrm{I}}$ gas fractions of AGN and non-AGN host galaxies.

\subsection{Gas fractions of AGN in the ALFALFA stacks}

We now turn to the ALFALFA spectral stacking analysis. In the top panel of Fig. 8 we show the $\mathrm{H}_{\mathrm{I}}$ gas fractions as a function of stellar mass for the AGN (star symbols) and non-AGN (circles) host ALFALFA spectral stacks. The numbers underneath the data points indicate how many individual spectra contributed to a given stack. The stellar mass range for each spectral stack is 0.3 dex, i.e. a mass bin width that is equivalent to the stellar mass matching criterion of \pm 0.15 dex used in the xGASS matching procedure. The exception is in the lowest stellar mass bin where a paucity of AGN necessitates a broader stellar mass range of 0.6 dex in order to achieve sufficient numbers and sensitivity in the stack. Each data point in Fig 8 is plotted at the centre of its mass bin (which is close to the mean mass in that bin), with the x-axis error bars showing the width of the bin in which the stack was constructed. In the lower panel of Fig. 8 we compute a gas fraction offset analogous to that computed for the individual xGASS galaxies. However, whereas in the xGASS analysis we were able to compute a gas fraction offset on a galaxy-by-galaxy basis, for the ALFALFA spectral stacks, we compute $\Delta f_{\text {gas }}$ as simply the difference of $\log f_{\text {gas }}$ for the AGN and non-AGN host stacks (i.e, the difference between star and circle symbols in the upper panel of Fig 8 .

The top panel of Fig. 8 shows that at stellar masses log $\left(\mathrm{M}_{\star} / \mathrm{M}_{\odot}\right)>10.2$ AGN hosts are more gas-rich than the control in given stellar mass bin. The bottom panel of the figure shows that the gas fraction enhancement is typically $\sim 0.25$ dex at these high masses. Although the error bars in Fig. 8 are quite large, the trend of elevated gas fractions at high stellar mass is systematic. Increasing the size of the stellar mass bins yields a more statisti- 

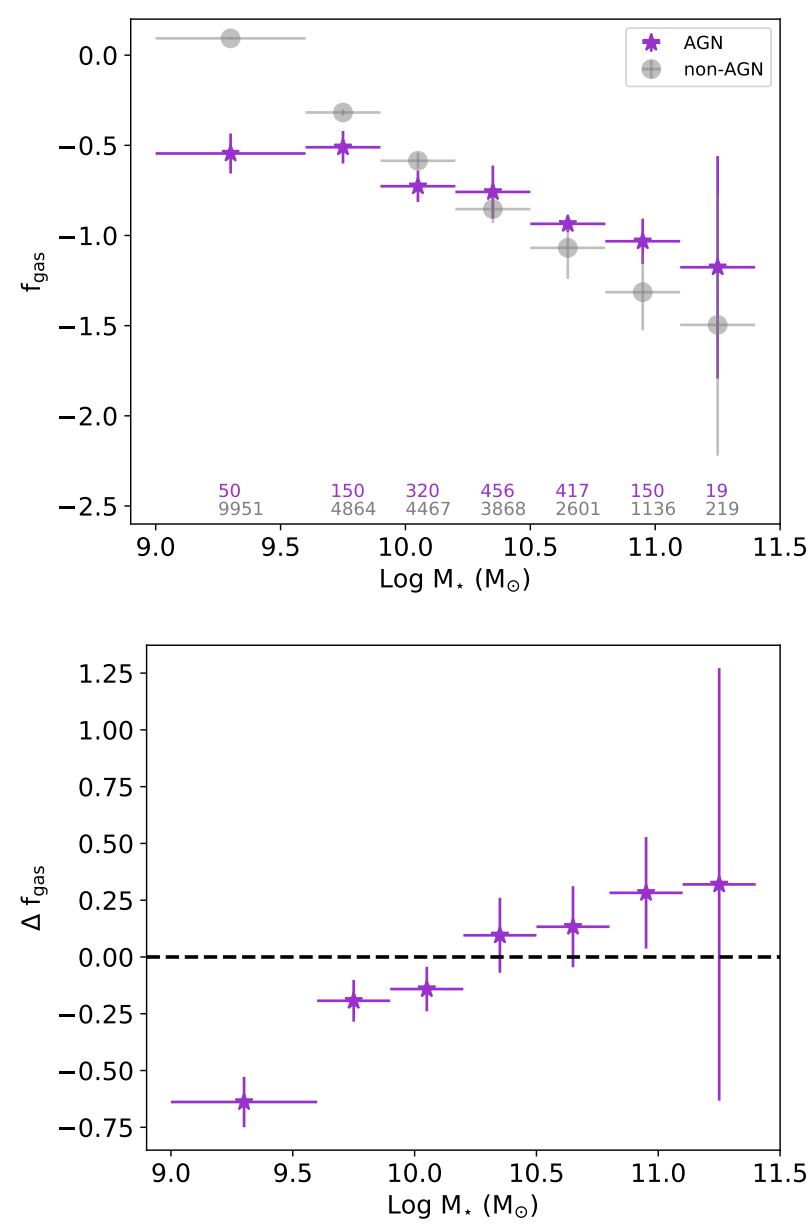

Figure 8. Top panel: Measured $f_{\text {gas }}$ for the AGN (purple stars) and nonAGN (grey circles) hosts in the ALFALFA spectral stacks in bins of stellar mass. Numbers under each data point indicate how many individual spectra contributed to each stack. Bottom panel: $\Delta f_{\text {gas }}$, computed as the difference between the AGN and non-AGN host stack gas fractions in the upper panel. For both panels, each data point is plotted at the centre of its mass bin (which is close to the mean mass in that bin), with the $\mathrm{x}$-axis error bars showing the width of the bin in which the stack was constructed.

cally significant result, although for consistency with the bins used in the xGASS analysis (which uses a mass matching tolerance of 0.15 dex) we have kept the original \pm 0.15 dex (i.e. 0.3 dex width) mass bins. The results at high stellar mass in Fig. 8 are broadly consistent with our finding of elevated $f_{\text {gas }}$ in individual xGASS AGN host galaxies, e.g. Fig. 5 which shows gas fraction enhancements above $\log \left(\mathrm{M}_{\star} / \mathrm{M}_{\odot}\right) \sim 10.2$ and with a sample median enhancement of $\Delta f_{\text {gas }}=0.29$ dex $($ Fig 4$)$.

The larger size of the ALFALFA sample, compared to xGASS, means that we include AGN host galaxies down to lower stellar masses. Fig. 8 reveals that at $\log \left(\mathrm{M}_{\star} / \mathrm{M}_{\odot}\right)<10$, a regime not probed by the xGASS sample, the gas fractions of the AGN hosts are now lower than in the stellar-mass matched control sample. This is a statistically significant result, particularly for the lowest mass AGN host galaxies in our sample, $9<\log \left(\mathrm{M}_{\star} / \mathrm{M}_{\odot}\right)<9.6$ (a total of $50 \mathrm{AGN}$ ), which are a factor $\sim 4$ more $\mathrm{H}_{\mathrm{I}}$ poor than non-AGN hosts of the same stellar mass.

As for the xGASS analysis, we next investigate the additional impact of matching the control sample not only in stellar mass,

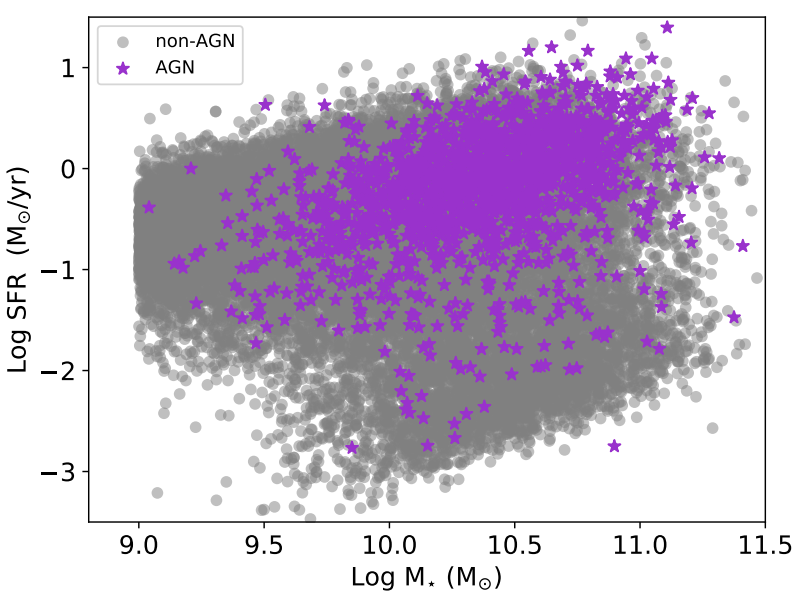

Figure 9. Distribution of SFR versus stellar mass for the ALFALFA sample. Non-AGN hosts are plotted with grey circles and AGN hosts in purple stars. AGN hosts tend to lie on the star forming main sequence or green valley with relatively few quenched hosts.

but also in SFR. The distribution of SFRs and stellar masses for the ALFALFA sample is shown in Fig. 9 As was the case in the xGASS sample (Fig. 6), AGN hosts in the ALFALFA sample are also primarily located on the star-forming main sequence or in the green valley, motivating the need to match in SFR.

Again, the ALFALFA spectral stacking requires a slightly different practical procedure than for the xGASS sample, in which individual AGN hosts are matched to controls. For every AGN galaxy that is included in a given stellar mass stack, we identify the nonAGN control galaxy in ALFALFA that is the closest simultaneous match in both $M_{\star}$ and SFR (again, SFRs are taken from the A2 catalog of Salim et al. 2018). Due to the large number of non-AGN galaxies in ALFALFA (27,116), the control sample can be very tightly matched in these quantities, with both the SFRs and $M_{\star}$ values typically matched to within 0.02 dex or less (which is about an order of magnitude less than the uncertainty in these values) of the AGN value. Nonetheless, due to the combination of well defined mass bins used in our spectral stacking (nominally spanning 0.3 dex), and the permitted tolerance of the SFR matching, the number of individual spectra (numbers beneath the data points in Fig. 10, that go into the AGN and non-AGN host stacks can differ by a few.

Fig. 10 is analogous to the data shown in Fig. 8 but now has the additional SFR matching included in the stacked data. The upper panel shows the $f_{\text {gas }}$ values in stacks binned by stellar mass for the AGN hosts (purple stars) and controls (grey circles). The lower panel of Fig. 10 shows $\Delta f_{\text {gas }}-$ the difference between the AGN hosts and control gas fractions. Fig. 10 shows that there is no longer any indication for an $\mathrm{H}_{\mathrm{I}}$ excess at $\log \left(\mathrm{M}_{\star} / \mathrm{M}_{\odot}\right)>10.2$, or in any other stellar mass regime. Therefore, as we previously concluded based on the xGASS analysis, the $\mathrm{H}_{\mathrm{I}}$ excess at fixed stellar mass is entirely a result of AGN hosts being preferentially located on the star-forming main sequence. Once this is accounted for by matching in SFR, both the xGASS and ALFALFA stacking analyses find the majority of AGN host galaxies to be H i normal. The possible exception to $\mathrm{H}$ I normalcy in AGN hosts remains in the lowest stellar mass bin probed by the ALFALFA stacking analysis. In the 9 $<\log \left(\mathrm{M}_{\star} / \mathrm{M}_{\odot}\right)<9.6$ stellar mass bin in the lower panel of Fig. 10 AGN hosts still appear to be $\mathrm{H}$ I poor by a factor of two (down from a factor of four at fixed stellar mass without SFR matching). 

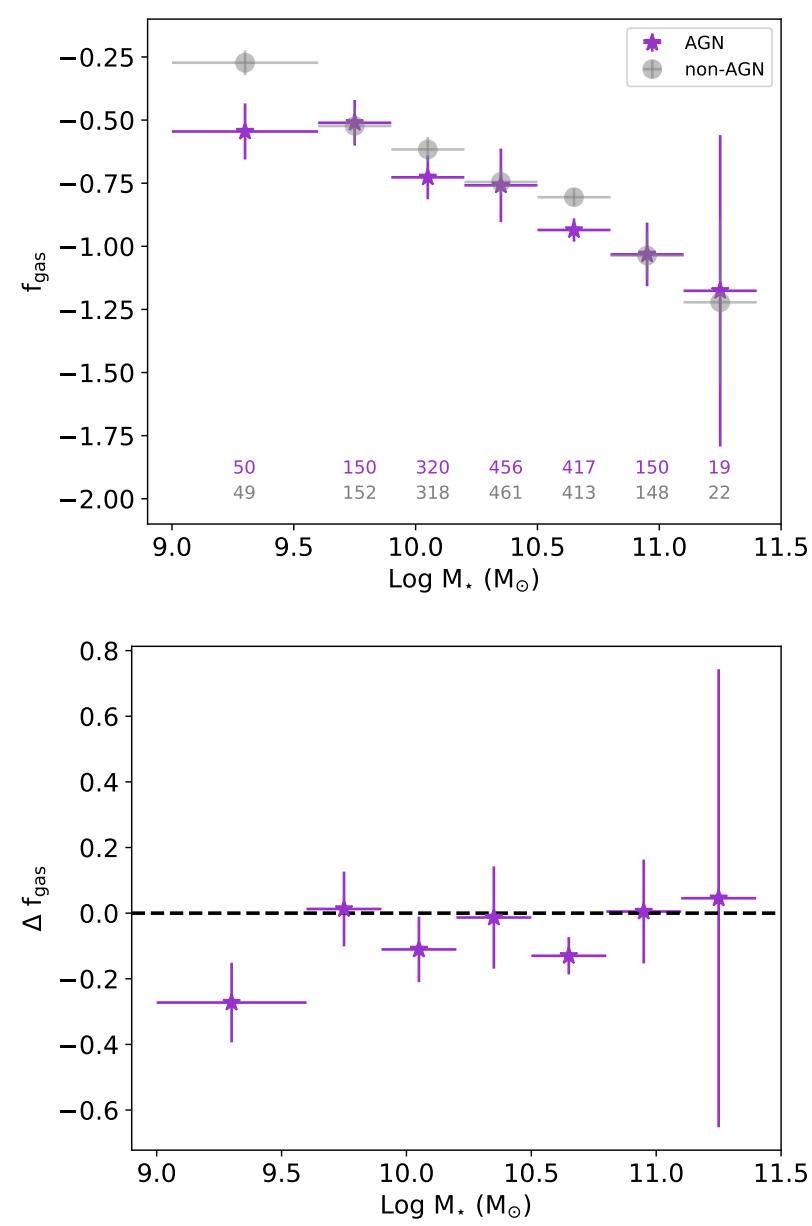

Figure 10. Top panel: Measured $f_{\text {gas }}$ for the AGN (purple stars) and nonAGN (grey circles) hosts in the ALFALFA spectral stacks in bins of stellar mass with control matching in both $M_{\star}$ and SFR. Numbers under each data point indicate how many individual spectra contributed to each stack. Bottom panel: $\Delta f_{\text {gas }}$, computed as the difference between the AGN and nonAGN host stack gas fractions in the upper panel. For both panels, each data point is plotted at the centre of its mass bin (which is close to the mean mass in that bin), with the $\mathrm{x}$-axis error bars showing the width of the bin in which the stack was constructed.

\section{DISCUSSION}

\subsection{Comparison to previous results - a reconciliation}

Previous comparisons of the $\mathrm{H}_{\mathrm{I}}$ content of AGN host galaxies that have used stacked $21 \mathrm{~cm}$ spectra have found no difference in average $f_{\text {gas }}$ compared with a non-AGN control sample (Fabello et al. 2011; Gereb et al. 2015). Likewise, using the relatively shallow ALFALFA survey to define 'normal' $\mathrm{H}_{\mathrm{I}}$ gas fractions, Bradford et al. (2018) have found no difference in atomic gas fractions in most AGN hosts. However, Bradford et al. (2018) report a possible deficit in $f_{\text {gas }}$ in AGN hosts for galaxies with $\log \left(\mathrm{M}_{\star} / \mathrm{M}_{\odot}\right)<9.5$. H I deficits in AGN host galaxies were also reported by Haan et al. (2008). Conversely, one of the earliest investigation of global $\mathrm{H}_{\mathrm{I}}$ gas fractions of AGN hosts, by Ho et al. (2008) reported the perhaps surprising result that AGN actually host higher gas fractions for their morphological type. Using the complementary technique of quasar absorption line spectroscopy to probe the circumgalactic medium, Berg et al. (2018) have also recently reported a factor of three excess Ly $\alpha$ absorption in sightlines through AGN hosts.

The literature has therefore variously reported normal, elevated and reduced $\mathrm{H}_{\mathrm{I}}$ gas fractions in and around AGN host galaxies. Different techniques, survey depths, assessment of comparison samples and treatment of limits likely contributes to the different conclusions drawn by previous works.

We have re-visited the quantification of $\mathrm{H}_{\mathrm{I}}$ gas fractions in optically selected AGN host galaxies in the SDSS using two complementary approaches: individual $f_{\text {gas }}$ measurements for a sample of 75 optically selected AGN for which we have H i measurements measured down to detection thresholds of only a few per cent, and spectral stacking for 1562 AGN in the ALFALFA survey. Both techniques take non-detections into account and use matched control samples for comparison drawn from the same datasets. Our experiment is therefore both well controlled for systematics, and uses two different techniques for cross-validation.

Both the individual $x$ GASS measurements and the ALFALFA stacks show that $\mathrm{AGN}$ hosts with $\log \left(\mathrm{M}_{\star} / \mathrm{M}_{\odot}\right) \gtrsim 10.2$ have $\mathrm{H}_{\mathrm{I}}$ gas fractions that are a factor of $\sim 2$ higher than non-AGN of the same stellar mass (Figs 4 and 8). Although the xGASS sample only contains AGN with $\log \left(\mathrm{M}_{\star} / \mathrm{M}_{\odot}\right)>10$, the ALFALFA stacks allow us to probe down to $\log \left(\mathrm{M}_{\star} / \mathrm{M}_{\odot}\right) \sim 9.0$. For AGN hosts in the ALFALFA sample with $\log \left(\mathrm{M}_{\star} / \mathrm{M}_{\odot}\right)<10$, we find a significant deficit of $\mathrm{HI}_{\mathrm{I}}$, at fixed stellar mass. This deficit is greatest in the lowest stellar mass bin, $9.0<\log \left(\mathrm{M}_{\star} / \mathrm{M}_{\odot}\right)<9.6$, where the $\mathrm{H}_{\mathrm{I}}$ gas fraction is lower than the non-AGN hosts by a factor of $\sim 4$ (Fig 8). The two complementary techniques used here therefore yield consistent results at high stellar masses (where the samples overlap and are comparable) and show that the gas fractions of AGN hosts are enhanced for their stellar mass.

However, a comparison between AGN and non-AGN hosts at fixed stellar mass fails to take into account that the former tend to inhabit star-forming or green valley galaxies, whereas the latter show a broad range of SFRs, including passive galaxies. We have shown that when this 'bias' is accounted for, by matching the nonAGN control sample in both stellar mass and SFR, the enhanced $f_{\text {gas }}$ in AGN hosts disappears (Figs. 7 and 10 . This is confirmed with both the xGASS and ALFALFA analyses. Therefore, conclusions concerning the gas fraction in AGN hosts depend not only on the stellar mass of the galaxy, but also on the experimental design and control sample parameters.

Our results help resolve several apparent inconsistencies that have been previously reported in the literature. For example, Ho et al. (2008) and Berg et al. (2018) have both reported H i excesses in AGN hosts. Both of these studies focused on relatively massive galaxies and matched either directly (Berg et al. 2018) or indirectly (using luminosity, Ho et al. 2008) on galaxy stellar mass. The results of both Ho et al. (2008) and Berg et al. (2018) are therefore in agreement with the factor of two $\mathrm{H}_{\mathrm{I}}$ excess for AGN host galaxies with $\log \left(\mathrm{M}_{\star} / \mathrm{M}_{\odot}\right) \gtrsim 10.2$ that we find in xGASS and ALFALFA (Figs 4 and 8 .

On the other hand, Fabello et al. (2011) and Gereb et al. (2015) found that AGN hosts are $\mathrm{H}$ i normal. Both of these studies take into account the $N U V-r$ colour of the AGN hosts and compare their $\mathrm{H}_{\mathrm{I}}$ gas fractions to controls of similar colours. This approach is similar to our comparison of $\mathrm{H}_{\mathrm{I}}$ gas fractions that are matched in both stellar mass and SFR. Therefore, our finding that matching in both of these parameters results in gas fractions that are consistent between AGN hosts and controls (Figs 7 and 10) is in good agreement with Fabello et al. (2011) and Gereb et al. (2015).

Finally, Bradford et al. (2018) found that AGN host galaxies 
with $\log \left(\mathrm{M}_{\star} / \mathrm{M}_{\odot}\right)<9.5$ are $\mathrm{H}_{\text {I }}$ poor. Again, this is consistent with our findings from the ALFALFA stacks, both at fixed $M_{\star}$ (where AGN hosts are a factor of 4 more $\mathrm{H}_{\mathrm{I}}$-poor than the controls: Fig 8 , and also with additional SFR matching (factor of 2: Fig 10).

\subsection{Dependence of gas fraction on [OIII] luminosity}

For galaxies whose emission lines are dominated by photoionization from the AGN, the [OIII] line luminosity can be used as an indicator of AGN luminosity (e.g. Kauffmann et al. 2003b). Contamination from star formation can be minimized by selecting AGN according to the Kewley et al. (2001) criteria. In the upper panel of Fig 11 we plot $\Delta f_{\text {gas }}$ versus [OIII] luminosity for individual galaxies in the xGASS sample identified as AGN using the Kewley et al. (2001) cut. We have not attempted to make a bolometric correction to the [OIII] luminosities as this represents a simple (but unknown) multiplicative factor for the x-axis, which does not impact any correlation. We find no trend between the gas fraction enhancement and [OIII] luminosity.

In the lower panel of Fig 11 we plot $\mathrm{H}_{\mathrm{I}}$ gas fraction versus [OIII] luminosity for ALFALFA spectral stacks that are now constructed in bins of $\mathrm{L}(\mathrm{O}[\mathrm{III}])$. Again, these stacks are made only for AGN identified as AGN using the Kewley et al. (2001) criteria. Since $\mathrm{L}(\mathrm{O}[\mathrm{III}]$ ) is not a relevant (in the sense of measuring nuclear accretion) quantity for non-AGN galaxies, there is no corresponding control stack, and therefore we can only look for variation in $f_{\text {gas }}$ and not $\Delta f_{\text {gas }}$ in the spectral stacks. Nonetheless, the results for the ALFALFA sample again indicate that there is no dependence of gas fraction on the AGN luminosity. Therefore, neither the xGASS, nor the ALFALFA results provide any evidence that the gas reservoir is systematically more affected by higher AGN luminosities. In a complementray work, Shangguan et al. (2018) have recently quantified the total gas fractions of low redshift quasars, whose luminosities are considerably greater than our Seyfert sample, and again found that the majority of the hosts retain high gas fractions.

\section{SUMMARY AND CONCLUSIONS}

AGN feedback has been proposed to lead to the cessation of star formation via the heating and/or removal of the galactic gas reservoir. However, previous measurements of the $\mathrm{H}_{\mathrm{I}}$ gas fraction in optically selected AGN hosts have found values largely consistent with the non-AGN population in massive galaxies (e.g. Fabello et al. 2011; Gereb et al. 2015; Bradford et al. 2018). Conversely, other works have found that AGN hosts may be in fact be relatively $\mathrm{HI}_{\mathrm{I}}$ rich (Ho et al. 2008; Berg et al. 2018).

In order to understand these apparently conflicting results, we re-visit the assessment of atomic gas fractions in AGN hosts using data from two complementary surveys, and using two different methods of analysis. First, we use a sample of 75 optically selected AGN from the relatively deep xGASS survey, and show that, at fixed stellar mass, the H I detection fraction is higher for AGN than non-AGN hosts. With a careful accounting for non-detections, we can compute gas fraction 'offsets' compared to a mass matched non-AGN sample for 50 of the AGN hosts with $10<\log \left(\mathrm{M}_{\star} / \mathrm{M}_{\odot}\right)$ $<10.8$. We find that AGN host $\mathrm{H}_{\mathrm{I}}$ gas fractions are elevated by a factor of two compared to non-AGN galaxies of the same stellar mass. However, we suggest that this effect is driven by the tendency of optical AGN to inhabit star forming host galaxies. When we additionally match our non-AGN sample in SFR, the median gas fraction of the AGN hosts is consistent with the controls. We
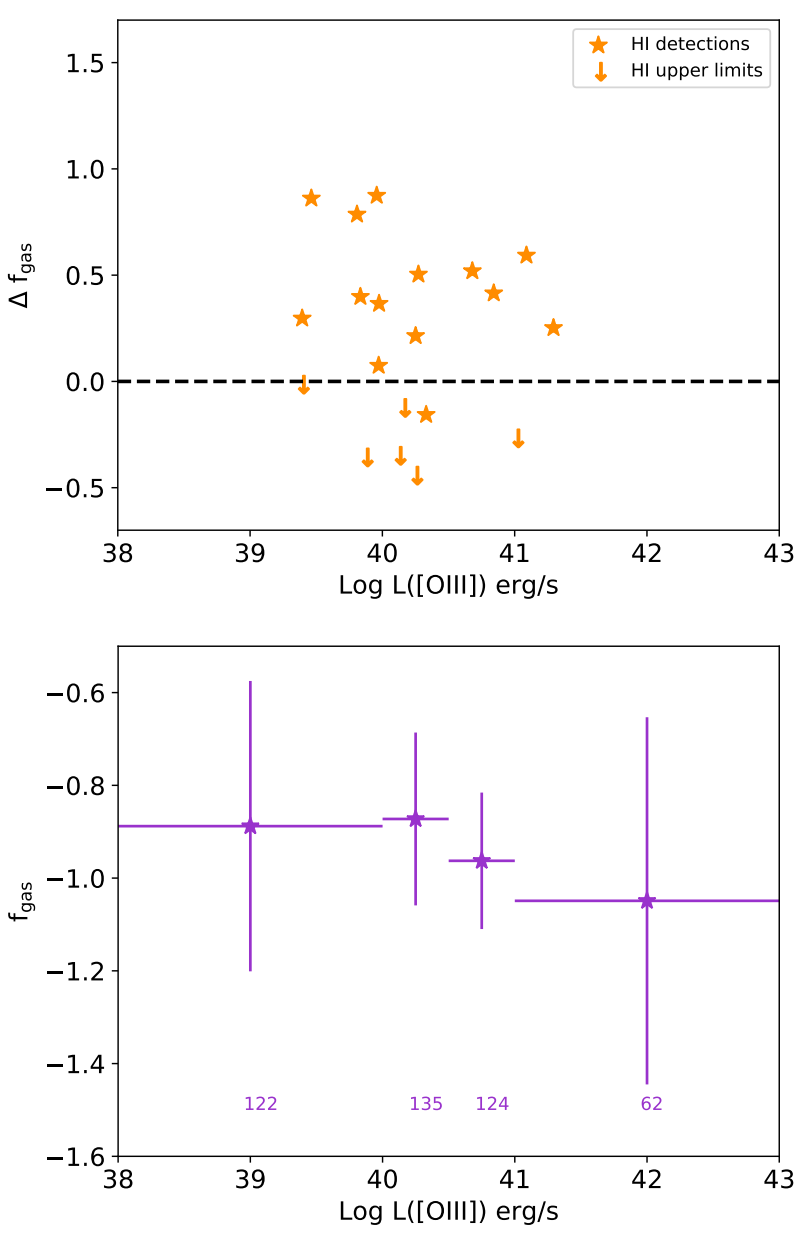

Figure 11. Top panel: $\Delta f_{\text {gas }}$ as a function of [OIII] line luminosity for AGN hosts in the xGASS sample. Lower panel: $f_{\text {gas }}$ as a function of [OIII] line luminosity for the ALFALFA stacks. [OIII] luminosities are only computed for galaxies classified as AGN by the Kewley et al. (2001) criterion, and have no bolometric correction applied. Bottom panel: $f_{\text {gas }}$ from ALFALFA stacks in bins of [OIII] line luminosity. Numbers under each data point indicate how many individual spectra contributed to each stack. Both panels are plotted on over the same range of [OIII] luminosity for ease of comparison.

conclude that in the regime $10<\log \left(\mathrm{M}_{\star} / \mathrm{M}_{\odot}\right)<10.8$ the gas fractions of optically selected AGN are consistent with non-AGN.

The second dataset studied in this work is a sample of $1562 \mathrm{op}-$ tically selected AGN from the 100 per cent ALFALFA survey. Although the ALFALFA survey is relatively shallow, spectral stacking permits an effective way to quantify gas fractions in sub-samples of the data and can take into account non-detections in individual spectra. We show that, in agreement with the xGASS results, $\mathrm{H}_{\mathrm{I}}$ gas fractions in AGN hosts are elevated by a factor of $\sim 2$ for $\log$ $\left(\mathrm{M}_{\star} / \mathrm{M}_{\odot}\right) \gtrsim 10.2$ at fixed stellar mass, compared with the non-AGN control sample. Also in agreement with the xGASS analysis, this excess disappears when the stacks are additionally matched in SFR. The ALFALFA stacking analysis therefore supports our conclusion from the xGASS analysis, that once properly matched in both $\mathrm{M}_{\star}$ and SFR, optically selected AGN in massive host galaxies are $\mathrm{H}_{\mathrm{I}}$ normal.

The larger size of the ALFALFA sample permits us to extend our analysis to lower stellar masses than is possible with the xGASS sample. We find that, at fixed stellar mass (with no SFR 
matching), AGN hosts with $\log \left(\mathrm{M}_{\star} / \mathrm{M}_{\odot}\right)<10.0$ are $\mathrm{H}_{\mathrm{I}}$ poor. For the lowest stellar masses, $9.0<\log \left(\mathrm{M}_{\star} / \mathrm{M}_{\odot}\right)<9.6$, this paucity is about a factor of 4 , and persists even when the SFR matching is implemented, albeit with lower magnitude (factor of two). The low gas fractions at low stellar mass are qualitatively consistent with the recent results of Bradford et al. (2018) and are consistent with reduction (either through removal or heating) of the atomic gas reservoir by AGN in the dwarf regime.

Our results help to reconcile apparently conflicting results in the literature, by showing that conclusions concerning the relative $\mathrm{H}$ I content of AGN hosts depends critically on how the control sample is constructed. In the context of AGN feedback and gas removal, we only find evidence of this process at the lowest stellar masses of our sample $\left(\log \left(\mathrm{M}_{\star} / \mathrm{M}_{\odot}\right)<10\right.$ at fixed stellar mass and $9<\log \left(\mathrm{M}_{\star} / \mathrm{M}_{\odot}\right)<9.6$ for fixed stellar mass and SFR). Moreover, neither the xGASS nor the ALFALFA AGN samples show any dependence of their gas fraction properties on AGN luminosity. Taken together, our results indicate that widespread removal of the host galaxy reservoir by the AGN is not a significant process for the majority of galaxies in the low redshift universe.

\section{ACKNOWLEDGEMENTS}

SLE gratefully acknowledges support from an NSERC Discovery Grant. Parts of this research were conducted by the Australian Research Council Centre of Excellence for All Sky Astrophysics in 3 Dimensions (ASTRO 3D), through project number CE170100013. We are grateful to Samir Salim for proving SDSS objIDs for the GSWLC and to Martha Haynes for providing access to the ALFALFA data cubes.

The xGASS and ALFALFA surveys, on which this work is based, were conducted at the Arecibo Observatory. The Arecibo Observatory is operated by SRI International under a cooperative agreement with the National Science Foundation (AST-1100968), and in alliance with Ana G. Méndez-Universidad Metropolitana, and the Universities Space Research Association.

Funding for the SDSS and SDSS-II has been provided by the Alfred P. Sloan Foundation, the Participating Institutions, the National Science Foundation, the U.S. Department of Energy, the National Aeronautics and Space Administration, the Japanese Monbukagakusho, the Max Planck Society, and the Higher Education Funding Council for England. The SDSS Web Site is http://www.sdss.org/

The SDSS is managed by the Astrophysical Research Consortium for the Participating Institutions. The Participating Institutions are the American Museum of Natural History, Astrophysical Institute Potsdam, University of Basel, University of Cambridge, Case Western Reserve University, University of Chicago, Drexel University, Fermilab, the Institute for Advanced Study, the Japan Participation Group, Johns Hopkins University, the Joint Institute for Nuclear Astrophysics, the Kavli Institute for Particle Astrophysics and Cosmology, the Korean Scientist Group, the Chinese Academy of Sciences (LAMOST), Los Alamos National Laboratory, the Max-Planck-Institute for Astronomy (MPIA), the MaxPlanck-Institute for Astrophysics (MPA), New Mexico State University, Ohio State University, University of Pittsburgh, University of Portsmouth, Princeton University, the United States Naval Observatory, and the University of Washington.

\section{REFERENCES}

Angles-Alcazar, D., Faucher-Giguere, C.-A., Keres, D., Hopkins, P. F., Quataert, E., Murray, N.,

Azadi, M., et al., 2017, ApJ, 835, 27

Berg, T. A. M., Ellison, S. L., Tumlinson, J., Oppenheimer, B., Horton, R., Bordoloi, R., Schaye, J., 2018, MNRAS, 4783890

Best, P. N., Heckman, T. M., 2012, MNRAS, 421, 1569

Bluck, A. F. L., et al., 2016, MNRAS, 462, 2559

Bluck, A. F. L., Mendel, J. T., Ellison, S. L., Moreno, J., Simard, L., Patton, D. R., Starkenburg, E., 2014, MNRAS, 441, 599

Bradford, J. D., Geha, M. C., Greene, J. E., Reines, A. E., Dickey, C. M., 2018, ApJ, 861, 50

Brown, T., Catinella, B., Cortese, L., Kilborn, V., Haynes, M. P., Giovanelli, R., 2015, MNRAS, 452, 2479

Catinella, B., et al, 2010, MNRAS, 403, 683

Catinella, B., et al, 2018, MNRAS, 476, 875

Cheung, E., et al., 2012, ApJ, 760, 131

Cicone, C., et al., 2014, A\&A, 562, 21

Concas, A., Popesso, P., Brusa, M., Mainieri, V., Erfanianfar, G.. Morselli, L., 2017, A\&A, 606, 36

Cowley, M. J., et al., 2016, MNRAS, 457, 629

Davies, R. L., et al., ApJ, submitted

Ellison, S. L., Catinella, B., Cortese, L., 2018, MNRAS, 438, 3447

Ellison, S. L., Fertig, D., Rosenberg, J. L., Nair, P., Simard, L., Torrey, P., Patton, D. R., 2015, MNRAS, 448, 221

Ellison, S. L., Teimoorinia, H., Rosario, D. J., Mendel, J. T., 2016a, MNRAS, 455, 370

Ellison, S. L., Teimoorinia, H., Rosario, D. J., Mendel, J. T., 2016b, MNRAS, 458, L34

Fabello, S., Kauffmann, G., Catinella, B., Giovanelli, R., Haynes, M. P., Heckman, T. M., Schiminovich, D., 2011, MNRAS, 416, 1739

Fang, J. J., Faber, S. M., Koo, D. C., Dekel, A., 2013, ApJ, 776, 63

Fluetsch, A., et al., 2018, MNRAS, submitted

Giovanelli, R., et al., 2005, AJ, 130, 2598

Haan, S., Schinnerer, E., Mundell, C. G., Garcia-Burillo, S., Combes, F., 2008, AJ, 135, 232

Harrison, C. M., Costa, T., Tadhunter, C. N., Flutsch, A., Kakkad, D., Perna, M., Vietri, G., 2018, NatAs, 2, 198

Haynes, M. P., et al., 2018, ApJ, 861, 49

Ho, L. C., Darling, J., Greene, J. E., 2008, ApJ, 681, 128

Hughes, T. M., \& Cortese, L., 2009, MNRAS, 396, L41

Husemann, B., Davis, T. A., Jahnke, K., Dannerbauer, H., Urrutia, T. Hodge, J., 2017, MNRAS, 470, 1570

Janowiecki, S., Catinella, B., Cortese, L., Saintonge, A., Brown, T., Wang, J., 2017, MNRAS, 466, 4795

Kauffmann, G., et al., 2003a, MNRAS, 341, 33

Kauffmann, G., et al., 2003b, MNRAS, 346, 1055

Kewley, L. J., Dopita, M. A., Sutherland, R. S., Heisler, C. A., Trevena, J., 2001, ApJ, 556, 121

Lang, P., et al., 2014, ApJ, 788, 11

Leslie, S. K., Kewley, L. J., Sanders, D. B., Lee, N., 2016, MNRAS, 455, 82

Lilly, S. J., \& Carollo, C. M., 2016, ApJ, 833, 1

McElroy, R., Croom, S. M., Pracy, M., Sharp, R., Ho, I.T., Medling, A. M., 2015, MNRAS, 446, 2186

Mendel, J. T., Simard, L., Ellison, S. L., Patton, D. R., 2013, MNRAS, 429, 2212

Mullaney, J. R., Alexander, D. M., Fine, S., Goulding, A. D., Harrison, C. M., Hickox, R. C., 2013, MNRAS, 433, 622

Muratov, A. L., Keres, D., Faucher-Giguere, C.-A., Hopkins, P. F., Quataert, E., Murray, N., 2015, MNRAS, 454, 2691

Omand, C. M. B., Balogh, M. L., Poggianti, B. M., 2014, MNRAS, 440, 843

Pereira-Santaella, M., et al., 2018, A\&A, 616, 171

Pei, Y., 1992, ApJ, 395, 130

Roberts-Borsani, G., \& Saintonge, A., 2018, MNRAS, in press

Rosario, D. J., et al., 2013, ApJ, 771, 63 
Rosario, D. J., Mendel, J. T., Ellison, S. L., Lutz, D., Trump, J. R., 2016, MNRAS, 457, 2703

Rosario, D. J., et al., 2018, MNRAS, 473, 5658

Saintonge, A., et al., 2012, ApJ, 758, 73

Salim, S., et al., 2016, ApJS, 227, 2

Salim, S., Boquien, M., Lee, J. C., 2018, ApJ, 859, 11

Santini, P., et al., 2012, A\&A, 540, 109

Scudder, J. M., Ellison, S. L., Mendel, J. T., 2012, MNRAS, 423, 2690

Shangguan, J., Ho, L. C., Xie, Y., 2018, ApJ, 854, 158

Shimizu, T. T., Mushotzky, R. F., Melendez, M., Koss, M., Rosario, D. J., 2015, MNRAS, 452, 1841

Teimoorinia, H., Bluck, A. F. L., \& Ellison, S. L., 2016, MNRAS, 457, 2086

Terrazas, B. A., Bell, E. F., Henriques, B. M. B., White, S. D. M., Cattaneo, A., Woo, J., 2016, ApJ, 830, L12

Woo, J.-H., Bae, H.-J., Son, D., Karouzos, M., 2016, ApJ, 817, 108

Woo, J., Dekel, A., Faber, S. M., Koo, D. C., 2015, MNRAS, 448, 237

Wright, E. L., et al., 2010, AJ, 140, 1868

Wuyts, S., et al., 2011, ApJ, 742, 96 